\title{
Almost Injective Mappings of Totally Bounded Metric Spaces into Finite Dimensional Euclidean Spaces
}

\author{
Gábor Sági ${ }^{1,2}$ \\ ${ }^{1}$ Hungarian Academy of Sciences, Alfréd Rényi Institute of Mathematics, Budapest, Hungary \\ ${ }^{2}$ Department of Algebra, Budapest University of Technology and Economics, Budapest, Hungary \\ Email: sagi@renyi.hu
}

How to cite this paper: Sági, G. (2019) Almost Injective Mappings of Totally Bounded Metric Spaces into Finite Dimensional Euclidean Spaces. Advances in Pure Mathematics, 9, 555-566.

https://doi.org/10.4236/apm.2019.96028

Received: May 25, 2019

Accepted: June 27, 2019

Published: June 30, 2019

Copyright () 2019 by author(s) and Scientific Research Publishing Inc. This work is licensed under the Creative Commons Attribution International License (CC BY 4.0).

http://creativecommons.org/licenses/by/4.0/

\section{Open Access}

\begin{abstract}
Let $\mathcal{X}=\langle X, \varrho\rangle$ be a metric space and let $\varepsilon$ be a positive real number. Then a function $f: X \rightarrow Y$ is defined to be an $\varepsilon$-map if and only if for all $y \in Y$, the diameter of $f^{-1}(y)$ is at most $\varepsilon$. In Theorem 10 we will give a new proof for the following well known fact: if $\mathcal{X}$ is totally bounded, then for all $\varepsilon$ there exists a finite number $n$ and a continuous $\varepsilon$-map $f_{\varepsilon}: X \rightarrow \mathbb{R}^{n}$ (here $\mathbb{R}^{n}$ is the usual $n$-dimensional Euclidean space endowed with the Euclidean metric). If $\varepsilon$ is "small", then $f_{\varepsilon}$ is "almost injective"; and still exists even if $\mathcal{X}$ has infinite covering dimension (in this case, $n$ depends on $\varepsilon$, of course). Contrary to the known proofs, our proof technique is effective in the sense, that it allows establishing estimations for $n$ in terms of $\varepsilon$ and structural properties of $\mathcal{X}$.
\end{abstract}

\section{Keywords}

Totally Bounded Metric Spaces, Dimension Theory, Finite Dimensional Euclidean Spaces, $\varepsilon$-Mapping

\section{Introduction}

It is a classical question in topology, that what kind of topological spaces $\mathcal{X}$ can be embedded into finite dimensional Euclidean spaces (endowed with the usual Euclidean topology). To motivate the present paper, first we recall some well known results in related investigations.

A classical characterization theorem can be recalled as follows. The covering dimension of $\mathcal{X}$ is finite if and only if there exists $n \in \mathbb{N}$ such that for every 
open covering $C$ of $\mathcal{X}$ there exists an open covering $C^{\prime}$ that refines $C$ and each point of $X$ belongs to at most $n+1$ elements of $C^{\prime}$ (the smallest $n$ satisfying this property is defined to be the dimension of $\mathcal{X}$; if there are no such $n$, then $\mathcal{X}$ is defined to be infinite dimensional). Clearly, if $\mathcal{X}$ can be embedded into a finite dimensional Euclidean space, then $\mathcal{X}$ is metrizable. By a classical theorem, for a compact, metrizable space $\mathcal{X}$ the followings are equivalent:

(1) $\mathcal{X}$ is finite dimensional (say, its dimension is $n$ );

(2) $\mathcal{X}$ is homeomorphic to a subspace of a finite dimensional (more concretely, $2 n+1$-dimensional) Euclidean space

(for a proof we refer to e.g. Corollary 50.9 of [1]). Recall, that for a positive real number $\varepsilon$, a function $f: X \rightarrow Y$ is defined to be an $\varepsilon$-map if and only if for all $y \in Y$ the diameter of $f^{-1}(y)$ is at most $\varepsilon$. Thus, if $\varepsilon$ is "small" then an $\varepsilon$-map is "almost injective". The usual proof for the harder direction of the above equivalence is based on showing that if the dimension of $\mathcal{X}$ is $n \in \mathbb{N}$, then for all $\varepsilon$, the set of continuous $\varepsilon$-mappings of $\mathcal{X}$ into $\mathbb{R}^{2 n+1}$ is dense and open; then the Baire category theorem implies the existence of a continuous embedding of $\mathcal{X}$ into $\mathbb{R}^{2 n+1}$.

Further, as explained in Theorem 2.2 of [2], for a compact, metrizable space $\mathcal{X}$, the above (1) and (2) are also equivalent with

(3) for all positive $\varepsilon, \mathcal{X}$ admits an $\varepsilon$-map to an $n$-dimensional simplicial complex.

In fact, Theorem 2.2 of [2] provides several statements equivalent with the above (1), (2) and (3) and recalls further notions of dimensions of topological spaces. All of these dimensions are equivalent for compact metric spaces. For further details on related notions and results we refer to the rather comprehensive survey article [2] and to the references therein.

Recall e.g. from [3], that the metric space $\mathcal{X}$ is defined to be totally bounded if and only if for all positive $\varepsilon \in \mathbb{R}$ there exists a finite collection of $\varepsilon$-balls of $\mathcal{X}$ that covers $X$. Further, $\mathcal{X}$ is compact if and only if it is totally bounded and complete (that is, every Cauchy sequence of $\mathcal{X}$ is convergent).

The main result of the present paper is Theorem 10 where we show, that

(i) if $\mathcal{X}$ is totally bounded, then for all $\varepsilon>0$, there exists a continuous $\varepsilon$ -mapping of $\mathcal{X}$ into a finite dimensional Euclidean space $\mathbb{R}^{n}$, further

(ii) estimations for $n$ are also provided in terms of $\varepsilon$ and structural properties of $\mathcal{X}$.

We should make the following remarks: item (i) above is well known. Thus, "almost injective" functions still exist, even if $\mathcal{X}$ is infinite dimensional-of course, if $\mathcal{X}$ is infinite dimensional, then $n$ depends on $\varepsilon$. The known proofs are existential, they cannot provide any upper bound for $n$. However our proof for (i) is effective in the sense, that (as stated in (ii) above), based on it, one can establish estimations on $n$ in terms of $\varepsilon$ and structural properties of $\mathcal{X}$.

The structure of the paper is rather simple: we close this section by fixing our notation and Section 2 contains the proofs. As we mentioned, our main goal is 
to prove Theorem 10 .

\section{Notation}

Our notation is mostly standard, but the following list may help.

Throughout $\mathbb{N}$ denotes the set of natural numbers. In addition, $\mathbb{R}$ and $\mathbb{R}^{+}$ denotes the set of real numbers, and the set of positive real numbers, respectively.

Let $\mathcal{X}=\langle X, \varrho\rangle$ be a metric space, $a \in X$ and let $\gamma$ be a non-negative real number. As usual, the open $\gamma$-ball $B(\gamma, a)$ at $a$ is the set

$$
B(\gamma, a)=\{x \in X: \varrho(a, x)<\gamma\} \text {. }
$$

\section{Proofs}

Definition 1 A family $\left\{B_{i}: i \in I\right\}$ of $\gamma$-balls is defined to be a $\gamma$-net if and only if it covers $X$, that is,

$$
X=\bigcup_{i \in I} B_{i}
$$

Thus, $\mathcal{X}$ is a completely bounded metric space if and only if for all positive $\gamma \in \mathbb{R}$ there exists a finite $\gamma$-net in $\mathcal{X}$. In addition, as it is well known, $\mathcal{X}$ is compact if and only if it's metric is totally bounded and complete (i.e. every Cauchy sequence is convergent in $\mathcal{X}$ ). For further details we refer to [3], as well.

If $\mathcal{X}$ is a completely bounded metric space, then $v(\mathcal{X}, \gamma)$ denotes the smallest cardinality $\kappa$ for which there exists a $\kappa$-sized $\gamma$-net of $\mathcal{X}$.

Definition 2 Let $\mathcal{X}=\langle X, \varrho\rangle$ be a metric space, let $A \subseteq X$ and let $b \in X$. Then the $\Delta$-type of $b$ over $A$ in $\mathcal{X}$ is defined to be the function $t p_{\Delta}^{\mathcal{X}}(b / A): A \rightarrow \mathbb{R}$, such that for all $a \in A$ we have

$$
t p_{\Delta}^{\mathcal{X}}(b / A)(a)=\varrho(a, b) .
$$

Thus, the $\Delta$-type of $b$ is just the function describing the distances of $b$ from elements of $A$.

By a $\Delta$-type over $A$ we mean a function $p: A \rightarrow \mathbb{R}$ which is of the form $t p_{\Delta}^{\mathcal{X}}(b / A)$ for some $b \in X$.

Keeping the notation introduced so far, we say, that $c \in X$ realizes the $\Delta$ -type $p$ in $\mathcal{X}$ if and only if $p=t p^{\mathcal{X}}(c / A)$.

Remark 3 We motivate and explain the above terminology as follows. Let $\mathcal{X}=\langle X, \varrho\rangle$ be a metric space. One can associate a relational structure to $\mathcal{X}$ in the following way. If $\mathrm{d}$ is a distance of $\mathcal{X}$, that is, $d \in \operatorname{ran}(\varrho)$ then the binary relation $R_{d}$ is defined to be

$$
R_{d}=\left\{\langle a, b\rangle \in X^{2}: \varrho(a, b) \leq d\right\} .
$$

Thus, the relational structure $\left\langle X, R_{d}\right\rangle_{d \in \operatorname{ran}(\varrho)}$ completely describes $\mathcal{X}$ and, at the same time, it can be treated as a model for an appropriate first order language. Then our definition of $\Delta$-types is essentially the same, as atomic types of the first order relational structure $\left\langle X, R_{d}\right\rangle_{d \in \operatorname{ran}(\varrho)}$ in the usual, model theoretic 
sense.

Lemma 1 Let $\mathcal{X}=\langle X, \varrho\rangle$ be a metric space, let $A \subseteq X$ be finite and let $n=|A|$. Then for the function $f: X \rightarrow \mathbb{R}^{n}, f(x)=t p_{\Delta}^{\mathcal{X}}(x / A)$ we have

$$
(\forall x, y \in X)(\|f(x)-f(y)\| \leq \sqrt{n} \varrho(x, y)),
$$

particularly, $f$ is continuous.

Proof. Enumerate $A=\left\{a_{0}, \cdots, a_{n-1}\right\}$ and for each $i<n$ let $f_{i}: X \rightarrow \mathbb{R}$, $f_{i}(x)=\varrho\left(a_{i}, x\right)$. By a slight abuse of notation, we have

$$
f(x)=t p_{\Delta}^{\mathcal{X}}(x / A)=\left\langle f_{0}(x), \cdots, f_{n-1}(x)\right\rangle
$$

for all $x \in X$.

Let $i<n$ be fixed in this paragraph. Then for any $x, y \in X$ the triangle inequality yields

$$
\varrho\left(a_{i}, x\right) \leq \varrho\left(a_{i}, y\right)+\varrho(y, x)
$$

so we have

$$
\varrho\left(a_{i}, x\right)-\varrho\left(a_{i}, y\right) \leq \varrho(y, x) .
$$

Similarly, interchanging $x$ and $y$ in the previous estimations, we obtain

$$
\varrho\left(a_{i}, y\right)-\varrho\left(a_{i}, x\right) \leq \varrho(x, y)
$$

and hence

$$
\left|f_{i}(x)-f_{i}(y)\right|=\left|\varrho\left(a_{i}, x\right)-\varrho\left(a_{i}, y\right)\right| \leq \varrho(x, y) .
$$

Consequently, for all $x, y \in X$ we have

$$
\begin{aligned}
\|f(x)-f(y)\| & =\sqrt{\sum_{i=0}^{n-1}\left(f_{i}(x)-f_{i}(y)\right)^{2}} \stackrel{\text { by }(9)}{\leq} \sqrt{\sum_{i=0}^{n-1}(\rho(x, y))^{2}} \\
& =\sqrt{n(\varrho(x, y))^{2}}=\sqrt{n} \varrho(x, y),
\end{aligned}
$$

as desired.

The following notion is an approximate version of splitting introduced in [4].

Definition 4 Let $\mathcal{X}=\langle X, \varrho\rangle$ be a metric space, let $B \subseteq A \subseteq X$, let $p$ be a $\Delta$ -type over $A$ in $\mathcal{X}$ and let $\varepsilon, \delta$ be non-negative real numbers. Then we say, that $p$ is $(\varepsilon, \delta)$-splitting over $B$ if and only if there exist $c_{0}, c_{1} \in A$ such that for all $b \in B$ we have

$$
\left|\varrho\left(b, c_{0}\right)-\varrho\left(b, c_{1}\right)\right|<\delta
$$

but whenever a realizes $p$, we have

$$
\left|\varrho\left(a, c_{0}\right)-\varrho\left(a, c_{1}\right)\right| \geq \varepsilon .
$$

Keeping the notation introduced in the above definition, intuitively $p=t p_{\Delta}^{\mathcal{X}}(a / A)$ is $(\varepsilon, \delta)$-splitting over $B$ if and only if there exist $c_{0}, c_{1} \in A$ such that $c_{0}$ and $c_{1}$ are "indiscernible from the viewpoint of $B$ modulo $\delta$ ", but a "distinguishes them modulo $\varepsilon$ ".

The following theorems will be essential in this paper. Some variants of them (in different contexts) had been utilized e.g. in [5], [6] and in [7]. 
Theorem 5 Let $\mathcal{X}=\langle X, \varrho\rangle$ be a totally bounded metric space, let $a \in X$, let $\varepsilon \in \mathbb{R}^{+}$and let $0<\delta \leq \frac{\varepsilon}{5}$ be arbitrary. Suppose

$$
A_{0} \subseteq A_{1} \subseteq \cdots \subseteq A_{M}
$$

is a strictly increasing sequence of subsets of $X-\{a\}$ such that for all $n<M$ the type $t_{\Delta}^{\mathcal{X}}\left(a / A_{n+1}\right)$ is $(\varepsilon, \delta)$-splitting over $A_{n}$. Then $M \leq v(\mathcal{X}, \delta)$.

Proof. Let $\left\{B_{i}: i<v(\mathcal{X}, \delta)\right\}$ be a $\delta$-net of $\mathcal{X}$ with smallest possible cardinality. By our assumption on splitting, for each $n<M$ there exist $c_{n}, d_{n} \in A_{n+1}-A_{n}$ such that for all $b \in A_{n}$ we have

$$
\left|\varrho\left(b, c_{n}\right)-\varrho\left(b, d_{n}\right)\right|<\delta
$$

but

$$
\left|\varrho\left(a, c_{n}\right)-\varrho\left(a, d_{n}\right)\right| \geq \varepsilon .
$$

Assume, seeking a contradiction, that $M>v(\mathcal{X}, \delta)$. By the pigeonhole principle, there exists $N \leq v(\mathcal{X}, \delta)$ such that $B_{N}$ (the $N^{\text {th }} \delta$-ball in our net) contains at least two $c_{n}$ 's; more precisely, there exist $n_{0}<n_{1} \leq 1+v(\mathcal{X}, \delta)$ with $c_{n_{0}}, c_{n_{1}} \in B_{N}$. Since $B_{N}$ is a $\delta$-ball, it follows, that $\varrho\left(c_{n_{0}}, c_{n_{1}}\right)<2 \delta$. Therefore, by the triangle inequality,

$$
\varrho\left(a, c_{n_{1}}\right) \leq \varrho\left(a, c_{n_{0}}\right)+\varrho\left(c_{n_{0}}, c_{n_{1}}\right)<\varrho\left(a, c_{n_{0}}\right)+2 \delta,
$$

and by symmetry,

$$
\varrho\left(a, c_{n_{0}}\right)<\varrho\left(a, c_{n_{1}}\right)+2 \delta .
$$

It follows, that

$$
\left|\varrho\left(a, c_{n_{0}}\right)-\varrho\left(a, c_{n_{1}}\right)\right|<2 \delta .
$$

By construction,

$$
\left|\varrho\left(c_{n_{0}}, c_{n_{1}}\right)-\varrho\left(c_{n_{0}}, d_{n_{1}}\right)\right|<\delta,
$$

particularly,

$$
\varrho\left(c_{n_{0}}, d_{n_{1}}\right)<3 \delta
$$

Therefore,

$$
\varrho\left(a, c_{n_{0}}\right) \leq \varrho\left(a, d_{n_{1}}\right)+\varrho\left(d_{n_{1}}, c_{n_{0}}\right)<\varrho\left(a, d_{n_{1}}\right)+3 \delta
$$

and similarly,

$$
\varrho\left(a, d_{n_{1}}\right) \leq \varrho\left(a, c_{n_{0}}\right)+\varrho\left(c_{n_{0}}, d_{n_{1}}\right)<\varrho\left(a, c_{n_{0}}\right)+3 \delta .
$$

It follows, that

$$
\left|\varrho\left(a, c_{n_{0}}\right)-\varrho\left(a, d_{n_{1}}\right)\right|<3 \delta
$$

Combining these, we get

$$
\left|\varrho\left(a, c_{n_{1}}\right)-\varrho\left(a, d_{n_{1}}\right)\right| \leq\left|\varrho\left(a, c_{n_{1}}\right)-\varrho\left(a, c_{n_{0}}\right)\right|+\left|\varrho\left(a, c_{n_{0}}\right)-\varrho\left(a, d_{n_{1}}\right)\right|<5 \delta .
$$


Since $5 \delta \leq \varepsilon$, this contradicts to (15) above, and the proof is complete.

Definition 6 Let $\mathcal{X}=\langle X, \varrho\rangle$ be a metric space, let $a \in X$ and let $\varepsilon, \delta \in \mathbb{R}^{+}$. Then $A \subseteq X$ is defined to be an $(\varepsilon, \delta)$-basis for a if and only if for any $B \subseteq X-\{a\}$ with $A \subseteq B$, the type

$$
\operatorname{tp}^{\mathcal{X}}(a / B)
$$

does not $(\varepsilon, \delta)$-split over $A$.

\section{Remark 7}

1) Clearly, if $A$ is an $(\varepsilon, \delta)$-basis for $a$ and $\varepsilon \leq \gamma$, then $A$ is a $(\gamma, \delta)$-basis for a, as well.

2) In addition, if $A$ is an $(\varepsilon, \delta)$-basis for $a$ and $A \subseteq B \subseteq X$ then $B$ is an $(\varepsilon, \delta)$-basis for $a$, as well.

Theorem 8 Let $\mathcal{X}=\langle X, \varrho\rangle$ be a totally bounded metric space, let $a \in X$ and let $\varepsilon \in \mathbb{R}^{+}$. Then there exist $\delta \in \mathbb{R}^{+}$and $A(a) \subseteq X-\{a\}$ such that $A(a)$ is an $(\varepsilon, \delta)$-basis for a.

In fact, arbitrary $\delta \leq \frac{\varepsilon}{5}$ is suitable and $A(a)$ can be chosen so, that $|A(a)| \leq 2(v(\mathcal{X}, \delta))$ is satisfied, as well.

Proof. Let $\delta \leq \frac{\varepsilon}{5}$ be an arbitrary positive real number.

Suppose, seeking a contradiction, that the consequence of the theorem is not true. By recursion, we define finite subsets $A_{n} \subseteq X-\{a\}$ for every natural number $n$, such that the following stipulations are satisfied:

(i) $A_{n} \subseteq A_{n+1}$, in fact, $A_{n+1}-A_{n}=\left\{c_{n}, d_{n}\right\}$;

(ii) $t p_{\Delta}^{\mathcal{X}}\left(a / A_{n+1}\right)$ is $(\varepsilon, \delta)$-splitting over $A_{n}$.

Let $A_{0}=\varnothing$ and suppose $A_{m}$ has already been defined for all $m \leq n$ such that stipulations (i) and (ii) are satisfied. Then, by our indirect assumption, there exists $B \subseteq X-\{a\}$ with $A_{n} \subseteq B$ such that $t p_{\Delta}^{\mathcal{X}}(a / B)$ is $(\varepsilon, \delta)$-splitting over $A_{n}$. This means, that there exist $c_{n}, d_{n} \in B$ such that for all $b \in A_{n}$ we have

$$
\left|\varrho\left(b, c_{n}\right)-\varrho\left(b, d_{n}\right)\right|<\delta
$$

but

$$
\left|\varrho\left(a, c_{n}\right)-\varrho\left(a, d_{n}\right)\right| \geq \varepsilon
$$

Let $A_{n+1}=A_{n} \cup\left\{c_{n}, d_{n}\right\}$. Then stipulations (i), (ii) remain true. In this way, one can define $A_{n}, c_{n}, d_{n}$ for all $n \leq 1+v(\mathcal{X}, \delta)$; this contradicts to Theorem 5 . Thus, the proof is complete: $A(a)$ can be chosen to be some $A_{n}$ (note, that an inspection shows, that each $A_{n}$ has cardinality at most $2 n$ and $2 n \leq 2 v(\mathcal{X}, \delta))$.

Lemma 2 Let $\mathcal{X}=\langle X, \varrho\rangle$ be a totally bounded metric space, let $a_{0}, a_{1}, a_{2}, b \in X$ and let $\varepsilon, \delta \in \mathbb{R}^{+}$. If $A$ is an $\left(\frac{\varepsilon}{3}, \delta\right)$-basis for $a_{0}, a_{1}$ and $a_{2}$, moreover $a_{0}, a_{1}$ and $a_{2}$ are pairwise distinct and $\varrho\left(a_{i}, b\right)<\frac{\varepsilon}{3} \quad$ for all 
$i \in\{0,1,2\}$, then $A$ is an $(\varepsilon, \delta)$-basis for $b$.

Proof. Assume, seeking a contradiction, that $A$ is an $\left(\frac{\varepsilon}{3}, \delta\right)$-basis for $a_{0}, a_{1}$ and $a_{2}$, but not an $(\varepsilon, \delta)$-basis for $b$. Then there exist $c, d \in X-\{b\}$ such that for all $u \in A$ we have

$$
|\varrho(u, c)-\varrho(u, d)|<\delta
$$

but

$$
|\varrho(b, c)-\varrho(b, d)| \geq \varepsilon .
$$

By assumption, $a_{0}, a_{1}$ and $a_{2}$ are pairwise distinct. It follows, that there exists $i \in\{0,1,2\}$ such that $a_{i} \notin\{c, d\}$, that is, $c, d \in X-\left\{a_{i}\right\}$.

Observe, that

$$
\varrho\left(a_{i}, c\right) \leq \varrho\left(a_{i}, b\right)+\varrho(b, c)
$$

and hence

$$
\varrho\left(a_{i}, c\right)-\varrho(b, c) \leq \varrho\left(a_{i}, b\right)<\frac{\varepsilon}{3} .
$$

Similarly,

$$
\varrho(b, c) \leq \varrho\left(b, a_{i}\right)+\varrho\left(a_{i}, c\right)
$$

and hence

$$
\varrho(b, c)-\varrho\left(a_{i}, c\right) \leq \varrho\left(b, a_{i}\right)<\frac{\varepsilon}{3} .
$$

Combining these estimations, we get

$$
\left|\varrho\left(a_{i}, c\right)-\varrho(b, c)\right|<\frac{\varepsilon}{3} .
$$

Completely similarly, one also can conclude, that

$$
\left|\varrho\left(a_{i}, d\right)-\varrho(b, d)\right|<\frac{\varepsilon}{3} .
$$

Observe moreover, that $\left|\varrho\left(a_{i}, c\right)-\varrho\left(a_{i}, d\right)\right|<\frac{\varepsilon}{3}$ because $A$ is an $\left(\frac{\varepsilon}{3}, \delta\right)$ -basis for $a_{i}$. But then,

$$
\begin{aligned}
& |\varrho(b, c)-\varrho(b, d)| \\
& \leq\left|\varrho(b, c)-\varrho\left(a_{i}, c\right)\right|+\left|\varrho\left(a_{i}, c\right)-\varrho\left(a_{i}, d\right)\right|+\left|\varrho\left(a_{i}, d\right)-\varrho(b, d)\right| \\
& <3 \cdot \frac{\varepsilon}{3}
\end{aligned}
$$

contradicting to (28).

Theorem 9 Let $\mathcal{X}=\langle X, \varrho\rangle$ be a totally bounded metric space. Then for each $\varepsilon \in \mathbb{R}^{+}$there exists a finite set $A \subseteq X$ such that $A$ is an $\left(\varepsilon, \frac{\varepsilon}{15}\right)$-basis for all $a \in X$.

In fact, we have $|A| \leq 6 \cdot v\left(\mathcal{X}, \frac{\varepsilon}{15}\right) \cdot v\left(\mathcal{X}, \frac{\varepsilon}{6}\right)$. 
Proof. Let $C \subseteq X$ be an $\frac{\varepsilon}{6}$-net of $\mathcal{X}$ with $|C|=v\left(\mathcal{X}, \frac{\varepsilon}{6}\right)$. Let

$$
C_{0}=\left\{b \in C:\left|B\left(b, \frac{\varepsilon}{6}\right)-\{b\}\right| \leq 2\right\}
$$

and let $C_{1}=C-C_{0}$. Enumerate $C_{1}$ as $C_{1}=\left\{B\left(b_{i}, \frac{\varepsilon}{6}\right): i<\left|C_{1}\right|\right\}$. For each $i<\left|C_{1}\right|$ there exist pairwise different $a_{b_{i}, 0}, a_{b_{i}, 1}, a_{b_{i}, 2} \in B\left(b_{i}, \frac{\varepsilon}{6}\right)$. By Theorem 8 , for each $i<\left|C_{1}\right|$ and $j<3$ there exists an $\left(\frac{\varepsilon}{3}, \frac{\varepsilon}{15}\right)$-basis $A\left(a_{b_{i}, j}\right)$ for $a_{b_{i}, j}$ with $\left|A\left(a_{b_{i}, j}\right)\right| \leq 2 v\left(\mathcal{X}, \frac{\varepsilon}{15}\right)$. Similarly, again by Theorem 8 , for each $b \in C_{0}$ and for each $a \in B\left(b, \frac{\varepsilon}{6}\right)$ there exists an $\left(\frac{\varepsilon}{3}, \frac{\varepsilon}{15}\right)$-basis $A(a)$ for a with $|A(a)| \leq 2 v\left(\mathcal{X}, \frac{\varepsilon}{15}\right)$. Finally, let

$$
A=\bigcup_{b \in C_{0}, a \in B\left(b, \frac{\varepsilon}{6}\right)} A(a) \cup \bigcup_{i<\left|C_{1}\right|, j<3} A\left(a_{b_{i}, j}\right) .
$$

We claim, that $A$ satisfies the conclusion of the theorem. By construction, clearly

$$
|A| \leq 2 \cdot v\left(\mathcal{X}, \frac{\varepsilon}{15}\right) \cdot 3 \cdot v\left(\mathcal{X}, \frac{\varepsilon}{6}\right)
$$

as desired. In addition, let $b \in A$ be arbitrary. Then there exists $b^{\prime} \in C$ such that $b \in B\left(b^{\prime}, \frac{\varepsilon}{6}\right)$.

If $b^{\prime} \in C_{0}$ then by construction, $A$ contains an $\left(\frac{\varepsilon}{3}, \frac{\varepsilon}{15}\right)$-basis $A(b)$ for $b$, which is also an $\left(\varepsilon, \frac{\varepsilon}{15}\right)$-basis for $b$ because of Remark 7 (1). Hence, by Remark 7 (2), $A$ is an $\left(\varepsilon, \frac{\varepsilon}{15}\right)$-basis for $b$.

If $b^{\prime} \in C_{1}$, then $b^{\prime}=b_{i}$ for some $i<\left|C_{1}\right|$. By construction, for all $j<3$ we have $\varrho\left(b_{i}, a_{b_{i}, j}\right)<\frac{\varepsilon}{6}$. Therefore, by the triangle inequality, for all $j<3$ we have

$$
\varrho\left(b, a_{b_{i}, j}\right) \leq \varrho\left(b, b_{i}\right)+\varrho\left(b_{i}, a_{b_{i}, j}\right) \leq \frac{\varepsilon}{6}+\frac{\varepsilon}{6}=\frac{\varepsilon}{3} .
$$

Hence, by Lemma $2, \bigcup_{j<3} A\left(a_{b_{i}, j}\right)$ is an $\left(\varepsilon, \frac{\varepsilon}{15}\right)$-basis for $b$. Since $A \supseteq \bigcup_{j<3} A\left(a_{b_{i}, j}\right)$, it follows from Remark 7 (2), that $A$ is an $\left(\varepsilon, \frac{\varepsilon}{15}\right)$-basis for $b$, as well. Since $b$ was arbitrary, the proof is complete. 
Lemma 3 Let $\langle X, \varrho\rangle$ be a metric space and let $\varepsilon, \delta \in \mathbb{R}^{+}$be arbitrary. Assume $A \subseteq X$ is an $\left(\frac{\varepsilon}{2}, \delta\right)$-basis for all $a \in X$. If $x \in X$ is not isolated, then for all $y \in X$

$$
t p_{\Delta}^{\mathcal{X}}(x / A)=t p_{\Delta}^{\mathcal{X}}(y / A) \text { implies } \rho(x, y) \leq \varepsilon .
$$

Proof. Since $x$ is not an isolated point of $\mathcal{X}$, there exists $z \in B\left(x, \frac{\varepsilon}{4}\right)-\{x\}$. By assumption, $A$ is an $\left(\frac{\varepsilon}{2}, \delta\right)$-basis for $z$, hence

$$
|\varrho(z, x)-\varrho(z, y)|<\frac{\varepsilon}{2}
$$

But $\varrho(z, x)<\frac{\varepsilon}{4}$, so it follows, that $\varrho(z, y) \leq \frac{3 \varepsilon}{4}$. Hence, by the triangle inequality,

$$
\varrho(x, y) \leq \varrho(x, z)+\varrho(z, y)<\frac{\varepsilon}{4}+\frac{3 \varepsilon}{4}=\varepsilon,
$$

as desired.

Let $\mathcal{X}=\langle X, \varrho\rangle$ be a metric space, let $\varepsilon \in \mathbb{R}^{+}$and let $Y$ be any set. As we mentioned in the introduction, according to the terminology of e.g. [2], a function $f: X \rightarrow Y$ is defined to be an $\varepsilon$-map if and only if for all $y \in Y$, the diameter of $f^{-1}(y)$ is at most $\varepsilon$, or equivalently,

$$
\sup \{\rho(x, y): x, y \in X, f(x)=f(y)\} \leq \varepsilon .
$$

Now we are able to state and prove the main result of the paper: we give a new proof for the fact, that each totally bounded metric space admits a continuous $\varepsilon$-map into some finite dimensional Euclidean space $\mathbb{R}^{n}$ (endowed with the usual Euclidean metric). Further, we provide upper bounds for $n$.

Theorem 10 Let $\mathcal{X}=\langle X, \varrho\rangle$ be a totally bounded metric space, let $\varepsilon \in \mathbb{R}^{+}$ and let $N=6 \cdot v\left(\mathcal{X}, \frac{\varepsilon}{30}\right) \cdot v\left(\mathcal{X}, \frac{\varepsilon}{12}\right)$.

1) if $\mathcal{X}$ does not contain isolated points, then there exist $n \leq N$ and an $\varepsilon$ -map $f: X \rightarrow \mathbb{R}^{n}$ such that, for all $x, y \in X$ we have $\|f(x)-f(y)\| \leq \sqrt{n} \rho(x, y)$, particularly $f$ is continuous.

2) if $\mathcal{X}$ has countably many isolated points, then there exist $n \leq 1+N$ and a continuous $\varepsilon$-map $f: X \rightarrow \mathbb{R}^{n}$.

3) if $\mathcal{X}$ is compact, then there exist $n \leq 1+N$ and a continuous $\varepsilon$-map $f: X \rightarrow \mathbb{R}^{n}$.

Proof. First we show (1). By Theorem 9, there exists $A \subseteq X$ such that $A$ is an $\left(\frac{\varepsilon}{2}, \frac{\varepsilon}{30}\right)$-basis for all $a \in X$ and $|A| \leq N$. Let $n=|A|$ and let $f: X \rightarrow \mathbb{R}^{n}$,

$$
f(x)=t p_{\Delta}^{\mathcal{X}}(x / A)
$$

for all $x \in X$. Then, by Lemma 1 , for all $x, y \in X$ we have

$$
\|f(x)-f(y)\| \leq \sqrt{n} \rho(x, y) .
$$


Further, by assumption, $\mathcal{X}$ does not contain isolated points. Hence, by Lemma $3 \mathrm{f}$ is an $\varepsilon$-map, as desired.

To show (2), enumerate all the isolated points of $X$ as $\left\{p_{k}: k \in \mathbb{N}\right\}$. As in (1), by Theorem 9 there exists $A \subseteq X$ such that $A$ is an $\left(\frac{\varepsilon}{2}, \frac{\varepsilon}{30}\right)$-basis for all $a \in X$ and $|A| \leq N$. Let $n^{\prime}=|A|$ and let $f^{\prime}: X \rightarrow \mathbb{R}^{n}$,

$$
f^{\prime}(x)=t p_{\Delta}^{\mathcal{X}}(x / A)
$$

for all $x \in X$. Then, by Lemma 1 , for all $x, y \in X$ we have

$$
\left\|f^{\prime}(x)-f^{\prime}(y)\right\| \leq \sqrt{n^{\prime}} \rho(x, y) .
$$

Let $n=1+n^{\prime}$, let $h: \mathbb{R}^{n^{\prime}} \rightarrow \mathbb{R}^{n}$ be the function

$$
h\left(x_{0}, \cdots, x_{n^{\prime}-1}\right)=\left\langle x_{0}, \cdots, x_{n^{\prime}-1}, 0\right\rangle
$$

and let $f: X \rightarrow \mathbb{R}^{n}$,

$$
f(x)= \begin{cases}h\left(f^{\prime}(x)\right) & \text { if } x \text { is not an isolated point of } X, \\ h\left(f^{\prime}(x)\right)+\left\langle 0, \cdots, 0, \frac{1}{1+k}\right\rangle & \text { if } x=p_{k} .\end{cases}
$$

First we show, that $f$ is continuous. To do so, let $x \in X$ and let $\gamma \in \mathbb{R}^{+}$be arbitrary. We shall show, that there exists $\delta \in \mathbb{R}^{+}$such that for all $y \in B(x, \delta)$ we have $\|f(x)-f(y)\|<\gamma$.

Case 1: $x$ is isolated in $\mathcal{X}$. Then there exists $\delta \in \mathbb{R}^{+}$such that $\{x\}=B(x, \delta)$, hence, for all $y \in B(x, \delta)$ we have $\|f(x)-f(y)\|<\gamma$.

Case 2: $x$ is not isolated in $\mathcal{X}$. Then, by 45, there exists $\delta^{\prime}$ such that for all $y \in B\left(x, \delta^{\prime}\right)$ we have $\left\|f^{\prime}(x)-f^{\prime}(y)\right\|<\gamma / \sqrt{2}$. In addition, there exists $k \in \mathbb{N}$ with $\frac{1}{k+1}<\gamma / \sqrt{2}$. Choose $\delta^{\prime \prime} \in \mathbb{R}^{+}$such that if $l<k$ then $p_{l} \notin B\left(x, \delta^{\prime \prime \prime}\right)$ and finally let $\delta=\min \left\{\delta^{\prime}, \delta^{\prime \prime}\right\}$. Now let $y \in B(x, \delta)$.

If $y$ is not isolated, then

$$
\|f(x)-f(y)\|=\left\|f^{\prime}(x)-f^{\prime}(y)\right\|^{\text {by } \delta \leq \delta^{\prime}}<;
$$

If $y$ is isolated, then $y=p_{l}$ for some $l \geq k$ and hence

$$
\|f(x)-f(y)\| \leq \sqrt{\left\|f^{\prime}(x)-f^{\prime}(y)\right\|^{2}+\frac{1}{(l+1)^{2}}}<\sqrt{\frac{\gamma^{2}}{2}+\frac{\gamma^{2}}{2}}=\gamma,
$$

thus, $f$ is continuous, as desired.

Next we show, that $f$ is an $\varepsilon$-map. To do so, assume $x \neq y \in X$ are such, that $f(x)=f(y)$. Observe, that by construction, for any $k \in \mathbb{N}$, the $k^{\text {th }}$ isolated point $p_{k}$ is the unique element $z \in X$ such that the last coordinate of $f(z)$ is equal to $\frac{1}{k+1}$. Hence neither $x$ nor $y$ are isolated. Therefore

$$
0=\|f(x)-f(y)\|=\left\|f^{\prime}(x)-f^{\prime}(y)\right\|,
$$

hence $f^{\prime}(x)=f^{\prime}(y)$. Combining this with the definition of $f^{\prime}$, we obtain 
$t p_{\Delta}^{\mathcal{X}}(x / A)=t p_{\Delta}^{\mathcal{X}}(y / A)$. But then, Lemma 3 implies $\rho(x, y) \leq \varepsilon$.

To show (3), we note, that (e.g. by Corollary 4.1 .16 of [3]) a compact metric space is second countable, hence it may contain countably many isolated points, only. Thus, (2) implies (3).

\section{Concluding Remarks}

In Theorem 10 we have given a new proof for the fact, that each totally bounded metric space $\mathcal{X}$ admits a continuous $\varepsilon$-map into some finite dimensional Euclidean space $\mathbb{R}^{n}$ (endowed with the usual Euclidean metric). Further, we provided upper bounds for $n$ in terms of $\varepsilon$ and structural properties of $\mathcal{X}$. Our proof had been obtained as follows:

- As recalled in Remark 3, there is a well known method that associates a first order structure $\mathcal{A}(\mathcal{X})$ to a metric space $\mathcal{X}$;

- if $\mathcal{X}$ is totally bounded, then $\mathcal{A}(\mathcal{X})$ has nice properties inspired by (model theoretic) stability theory, more concretely, as shown in Theorem 5, if $\varepsilon \in \mathbb{R}^{+}$, then each increasing chain of $\left(\varepsilon, \frac{\varepsilon}{5}\right)$-splitting $\Delta$-types in $\mathcal{A}(\mathcal{X})$ should have finite length;

- as shown in Theorem 10, using the above observation one can construct an appropriate continuous $\varepsilon$-map from $\mathcal{X}$ to a finite dimensional Euclidean space.

The second item of the above list motivates the following problem which may serve as a starting point of a further investigation.

Open problem 11 Is the converse of Theorem 5 true, as well? That is, if $\mathcal{X}$ is a metric space such that, for all $\varepsilon \in \mathbb{R}^{+}$the length of any increasing chain of $\left(\varepsilon, \frac{\varepsilon}{5}\right)$-splitting $\Delta$-types in $\mathcal{A}(\mathcal{X})$ is finite, then does it follow, that $\mathcal{X}$ is totally bounded?

\section{Acknowledgements}

This work has been supported by Hungarian National Foundation for Scientific Research grant K129211.

\section{Conflicts of Interest}

The author declares no conflicts of interest regarding the publication of this paper.

\section{References}

[1] Munkres, J.R. (2000) Topology. Prentice Hall, Upper Saddle River.

[2] Dranishnikov, A.N. (2018) Dimension of Compact Metric Spaces. Indagationes Mathematicae, 29, 429-449. https://doi.org/10.1016/j.indag.2017.04.005

[3] Engelking, R. (1989) General Topology. Heldermann Verlag, Berlin.

[4] Shelah, S. (1990) Classification Theory. North-Holland, Amsterdam. 
[5] Sági, G. and Gyenis, Z. (2013) Upward Morley’s Theorem Downward. Mathematical Logic Quarterly, 59, 303-331. https://doi.org/10.1002/malq.201110048

[6] Sági, G. and Nyiri, D. (2016) On Embeddings of Finite Metric Spaces. The Proceedings of the 13th International Scientific Conference on Informatics, Poprad, 18-20 November 2015, 227-231. https://doi.org/10.1109/Informatics.2015.7377837

[7] Sági, G. (2015) Vaught's Conjecture from the Perspective of Algebraic Logic. Manuscript. 